\title{
Analysis of a Switched-Reluctance Generator for Maximum Energy Conversion
}

\author{
Supakit Wongguokoon Non-member (KMITL, supakitw@windowslive.com) \\ Supat Kittiratsatcha Non-member (KMITL, kksupat@kmitl.ac.th)
}

Keywords: analytical model, switched reluctance generator, converted energy, maximum energy conversion

Generation using switched-reluctance generators (SRGs) is carried out in many demanding applications, such as starter-alternator in automobiles, wind turbine applications, aerospace power system, and flywheel energy storage system, owing to their advantages of low cost, robustness, simplicity in machine assembly, high efficiency, and speed capability. An SRG has steel laminations on the rotor and stator. On the stator, coils are placed around each salient pole to form phase windings. On the other hand, there are no windings or permanent magnets on the rotor. In an SRG, the phase winding inductance depends on the rotor position. Generation in an SRG occurs in the $\mathrm{dL} / \mathrm{d} \theta<0$ zone.

Energy conversion in an SRG is a function of four control variables: DC-bus voltage, shaft speed, and turn-on and turn-off angles. For maximum energy conversion, experimental characterization of the target machine is required. The iterative measurement of the machine in terms of such control variables has been used to determine the energy conversion profile. The number of iterations is large owning to many control variables. Consequently, iteration measurement is time consuming and requires extensive experimental work.

This paper presents explicit equations for maximum energy conversion in terms of DC-bus voltage, shaft speed, and turn-on and turn-off angles. An analytical model is used to numerically describe the relationship between converted energy versus and the control variables. Optimal control variables for maximum energy conversion are then derived from the analytical model. The model is verified by comparing the experimental results and those obtained by the model.

Further, the analytical model describes the magetization curve of the machine, as shown in Fig. 1. It describes flux linkage $\psi$ depending on phase current $i$ and rotor position $\theta$. The model requires only three machine parameters: initial aligned inductance $L_{d}$, unaligned inductance $\mathrm{L}_{\mathrm{q}}$, and effective overlap angle $\theta_{\mathrm{k}}$, as shown by the following equation:

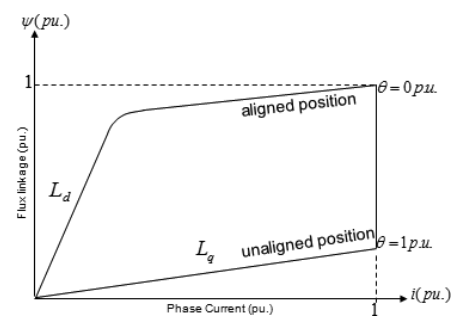

Fig. 1. Magnetization curve

$$
\psi(\mathrm{i}, \theta)=\mathrm{L}_{\mathrm{q}} \cdot \mathrm{i}+\frac{\left(\mathrm{L}_{\mathrm{d}}-\mathrm{L}_{\mathrm{q}}\right)}{1+\mathrm{k}_{\mathrm{s}} \cdot \mathrm{i}} \cdot \mathrm{i} \cdot \mathrm{f}(\theta)
$$

The converted energy $\mathrm{W}_{\text {net }}$ is the area enclosed by the $\psi$-i loci.

$$
\mathrm{W}_{\mathrm{net}}=\oint \psi \mathrm{di}
$$

From the results of the analysis, the converted energy versus the ratio $\mathrm{x}$ of the DC-bus voltage to shaft speed at different peak current levels is shown in Fig. 2.

Maximum energy conversion occurs at optimal ratio $\mathrm{x}_{\mathrm{opt}}$, which is obtained from the following equation.

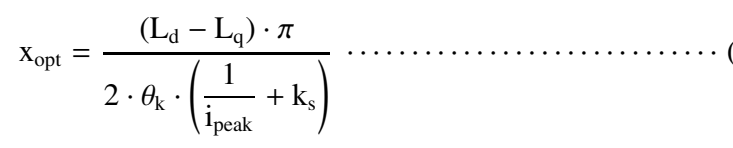

$x_{\text {opt }}$ is a function of the machine parameters and peak current $i_{\text {pk }}$. The DC-bus voltage and shaft speed are important control variables and their optimal ratio is obtained from (2) corresponding to a particular peak current. The peak current should reach its maximum value in order to utilize the maximum energy available. The crest factor is also improved when the conversion is optimal. Moreover, this implies that the utilization of inverter switch ratings are improved. The optimal turn-on angle is -0.5 p.u. for all values of $\mathrm{x}$. The turn-off angle is then adjusted to reach the given peak current. The converted energy is not very sensitive to variations in the turnon angle at its maximum value. Therefore, the control variable, excitation angle, can be used as a secondary control objective owning to which the converted energy is still closed to its maximum value. The experimental results for energy conversion for the control variables show the same trends as those for the analytical results.

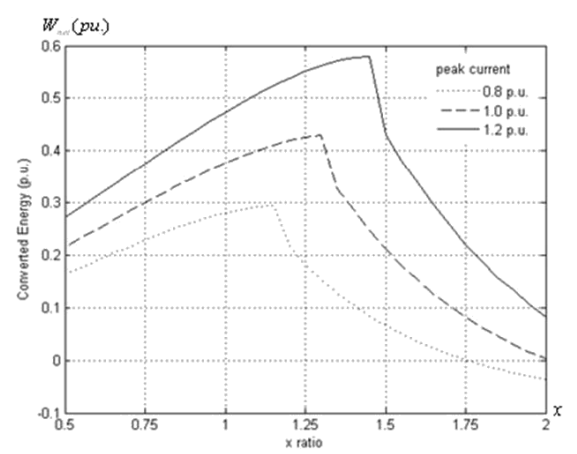

Fig. 2. $\mathrm{W}_{\text {net }}$ vs. ratio $\mathrm{x}$ at different peak current levels 


\title{
Analytical Modeling of Flat and Tubular Linear PM Machines with Surface-Mounted Magnets and Semi-Closed Slots
}

\author{
Georges Barakat Non-member (University of Le Havre, georges.barakat@univ-lehavre.fr) \\ Yacine Amara Non-member (University of Le Havre, yacine.amara@univ-lehavre.fr)
}

Keywords: linear machines, permanent magnet machines, analytical modeling, magnetic fields

This study attempts to provide analytical tools to facilitate the analysis and design of a class of linear PM machines (Fig. 1). A model is developed for giving the exact magnetic field distribution associated with permanent magnets and armature currents in surface-mounted flat and tubular linear machines by considering stator slotting. Compared to finite element model, the analytical model is less time-consuming and is more convenient to use in a design optimization process.

Indeed, analytical models of electric machines are often preferred in early design stages owing to the shorter time required compared to finite element models. Finite element analysis is always used for the verification and validation of designs obtained in the initial design stages. The verification and validation step is often effective when the analytical models used in the initial design stages are accurate and realistic. It is from this perspective that models presented in this paper have been developed.

The developed models embrace both linear machine structures: flat and tubular structures (Fig. 1). In the case of flat structures, the developed models can be used for single-sided and double-sided structures. For double-sided flat structures and tubular structures, the developed models cover both internal and external moving armature topologies. The armature supporting the permanent magnets can be made of magnetic or non-magnetic material for all structures.

The slotted stator has a classical configuration with straight teeth and semi-closed slots. The slots and teeth may or may not be equally distributed. They can be arranged to accommodate any winding configuration. Results from this analytical model are compared to those from finite element analyses.

The analytical solution allows the prediction of the main parameters and performances (EMF, cogging force, thrust force ....etc.) of the machines in closed forms, and it facilitates the evaluation of any possible partial, irreversible demagnetization of the magnets. It can also be used to estimate resistance-limited eddy current losses in permanent magnets and armature windings.

Figure 2 shows the different regions (stator slots (I), slot opening

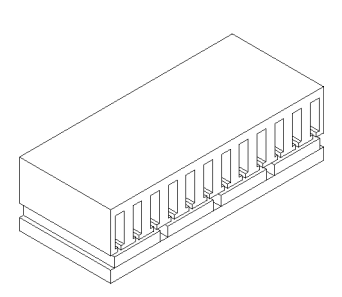

(a)

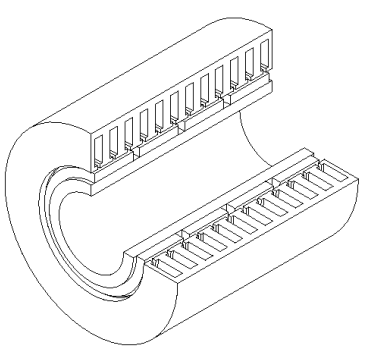

(b)
Fig. 1. Linear machine structures: flat (a) and tubular (b)
(II), air gap (III), and permanent magnets (IV)) where the exact analytical solution is determined by the separation-of-variable method. The model is set in 2D Cartesian coordinates $(x, y)$ for flat structures and 2D Polar coordinates $(r, z)$ for tubular structures. The analytical solution for the magnetic field distribution is obtained assuming that (1) the stator and moving cores are assumed to be infinitely permeable (magnetic saturation is neglected), (2) the permeability of permanent magnets is assumed to be equal to that of air, and (3) the axial length of the machine is infinite, which implies that the longitudinal end effects can be neglected. Transverse edge effects are also neglected for flat linear structures. The partial differential equation for quasi-stationary magnetic fields in a continuous and isotropic region can be expressed in terms of the magnetic vector potential $\boldsymbol{A}$, which is subjected to the Coulomb gauge $\nabla \cdot \boldsymbol{A}=0$ as

$$
\left\{\begin{aligned}
\nabla^{2} \boldsymbol{A} & =-\mu_{0} \boldsymbol{J} & & \text { in region I } \\
\nabla^{2} \boldsymbol{A} & =0 & & \text { in regions II and III }, \\
\nabla^{2} \boldsymbol{A} & =-\mu_{0} \nabla \times \boldsymbol{M} & & \text { in region IV }
\end{aligned}\right.
$$

where $\boldsymbol{M}$ is the remanent magnetization and $\boldsymbol{J}$ is the current density vector.

To show the efficiency of the analytical approach, in Fig. 3, magnetic field components for a flat linear machine obtained by both finite element (FE) and analytical models for the air gap region are compared. FE analysis is performed considering a very high relative permeability for the stator and moving cores.

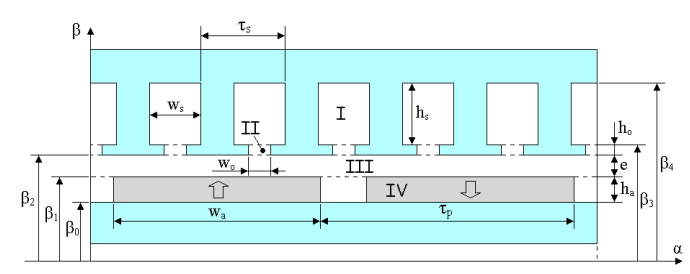

Fig. 2. Field regions in idealized linear machines

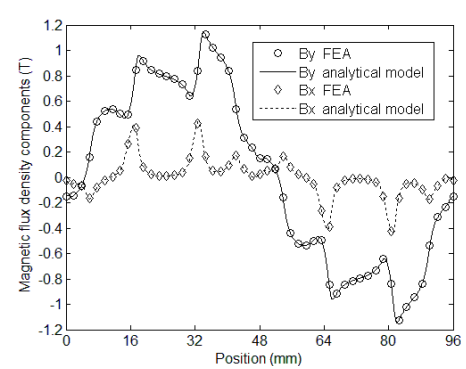

Fig. 3. Comparison of magnetic field components in region III (air-gap region) 


\title{
Frequency Response Analysis Considering Nonlinear Distribution of Complex Magnetic Permeability and Employing 3D FEM
}

\author{
Seung-Ho Yang Student Member (Osaka University) \\ Katsuhiro Hirata Senior Member (Osaka University) \\ Tomohiro Ota Member (Panasonic Electric Works, Ltd.) \\ Yoshihiro Kawase Senior Member (Gifu University)
}

Keywords: complex magnetic permeability, finite element method, frequency response analysis, nonlinear analysis

Frequency response analysis (FRA) is conventionally used for linear calculations. We have been studying a computational method for analyzing the characteristics of a linear position sensor and its core material; the method involves the use of FRA and also takes into account the complex magnetic permeability (CMP).

The magnetic permeability of the magnetic material that is used in the conventional FRA method has a fixed value. At high frequencies, the magnetic flux is concentrated near the surface of the material owing the skin effect. However, the magnetic flux density is actually distributed nonlinearly at the core of the material. Thus, the magnetic permeability reaches a high value near the surface and a low value inside the material. Therefore, the FRA approach needs to be revised to account for both the linear variation of the magnetic flux density with respect to time and the nonlinear distribution of the CMP with respect to the magnetic flux density within the material.

There have been a few papers in which analyses were conducted using CMP. However, we were able to find just one paper in which the concept of nonlinear CMP was considered.

In this paper, we propose a revised FRA method that takes into account the nonlinearity of the CMP. Moreover, in order to confirm the validity of this computation method, we compare the analyzed results (before and after considering the nonlinearity of the CMP) with the measurements obtained by using a prototype.

We determine the frequency characteristics of the CMP by varying the frequency from $30 \mathrm{~Hz}$ to $100 \mathrm{kHz}$. The skin effect greatly influences the magnetic flux distribution, especially at high frequencies. Therefore, we prepare a fine finite-element mesh around the core surface to consider the skin effect. In the analysis method, it was sufficient to consider $1 / 720$ of the surface area of the model owing to the symmetry of the model. The 3D mesh of the analyzed model of a toroidal coil is shown in Fig. 1.

We analyzed the frequency characteristics of the CMP of the core material, SUY. For the cases where the nonlinearity of the CMP was considered and not considered. The results are shown in Figs. 2 $\left(B_{\text {aver. }}=5 \mathrm{mT}\right)$ and $3\left(B_{\text {aver. }}=50 \mathrm{mT}\right)$.

We evaluated these results in terms of the difference in the real part of the CMP between the surface and the core of the material at the frequency of $1 \mathrm{kHz}$.

In the first case $(5 \mathrm{mT})$, the real part of the CMP near the surface is about three times larger than the real part of the CMP in the core. On the other hand, in the second case $(50 \mathrm{mT})$, the real parts of the CMP differ by a factor of about 10 . Thus, the effect of nonlinearity of the CMP is prominent in this analysis result.

It is clear that the larger the skin effect, the more advantageous is the proposed method compared with linear analysis.

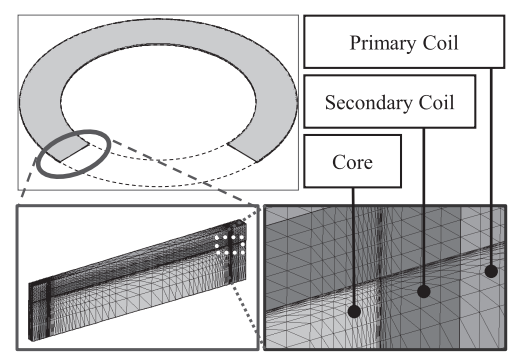

Fig. 1. Three-dimensional mesh of analyzed model (1 ${ }^{\circ}$ model)

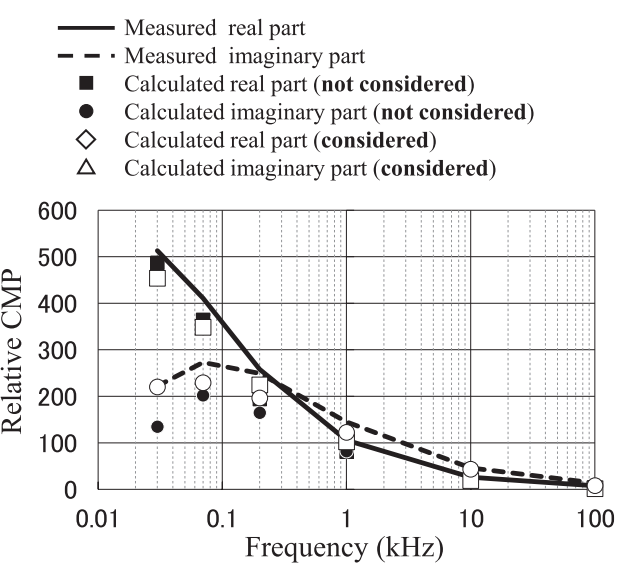

Fig. 2. Comparison between analyzed and measured results of frequency characteristics of complex magnetic permeability $\left(\mathrm{SUY}, B_{\text {aver }}=5 \mathrm{mT}\right)$

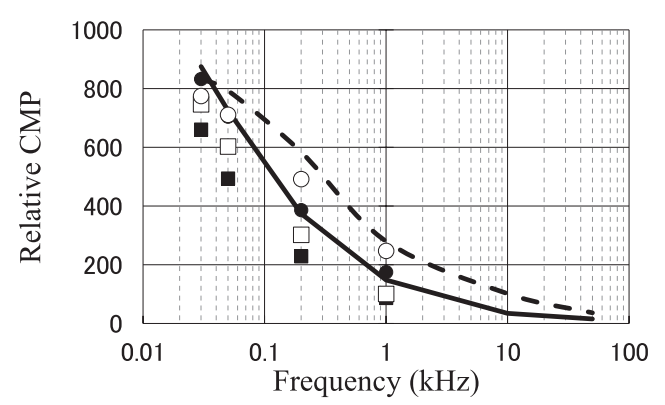

Fig. 3. Comparison between analyzed and measured results of frequency characteristics of complex magnetic permeability (SUY, $B_{\text {aver. }}=50 \mathrm{mT}$ ) 


\title{
Emerging Technologies for Multilevel Converters in Japan
}

\author{
Hideaki Fujita Member (Tokyo Institute of Technology)
}

Keywords: capacitor-clamped converters, diode-clamped converters, modular multilevel cascaded converters, multilevel power converters

This paper summarizes the multilevel power conversion technologies published in the the IEEJ Transactions on Industry ApPLicaTIONS and presented at IEEJ Technical Meeting on Semiconductor Power Converter or Japan Industry Applications Society Conference (JIASC) as well as international conference proceedings and journals.

Thirty years ago, the first neutral-point clamped (NPC) PWM converter was invented by Prof. Nabae. The NPC converter has capability of synthesizing the three-level output voltage, resulting in increase of the output voltage amplitude and in reduction of harmonic voltages. Today, the NPC converter have been put into practical applications, such as 3.3-kV industrial adjustable-speed motordrive systems, Nozomi 700 super express trains, and so on. The concept of the NPC converter has been expanded to multilevel power conversion circuits. Recently, many researchers and engineers have been working on multilevel converters capable of synthesizing voltage waveforms with five or more levels.

The multilevel converters are classified into two families: classical clamped converters and modular multilevel cascaded converters. Fig. 1 shows circuit configuration of clamped five-level converters. The diode-clamped converter (a) consists of series-connected switching devices, clamping diodes, and split dc capacitors. When the converter acts as an inverter, dc capacitors $C_{1}$ and $C_{4}$ are charged and $C_{2}$ and $C_{3}$ are discharged, and thus, an additional circuit is needed to balance the dc capacitor voltage. On the other hand, the capacitor clamped converter (b) has the capability to self balancing of the dc capacitor voltage, and does not require any additional voltage balancing circuit.

Various circuit topology are included in the modular multilevel cascaded converter family. Fig. 2 shows a single-star bridge-cell configuration, consisting of series-connected H-bridge converters. On the other hand, Fig. 3 shows a double-star chopper-cell configuration, where di-directional chopper circuits are connected in series. The most significant difference between two configurations is existence of the "dc link." Fig. 2 can not control the average active power, whereas Fig. 3 has the capability of converting active power from its dc side to the ac side. Therefore, Fig. 2 is expected to apply to STATCOMs or active power filters, and Fig. 3 would be better choice for medium-voltage motor drives or high-voltage dc transmission systems.

Recently-proposed active-clamped multilevel converters and current-source multilevel converters are presented along with their appropriate applications. This paper also describes some special circuit topologies based on cascaded H-bridge inverters and cascaded bi-directional chopper circuits having unequal dc capacitor voltages. Form these observations, effectiveness of multilevel converters in low voltage applications are finally discussed.
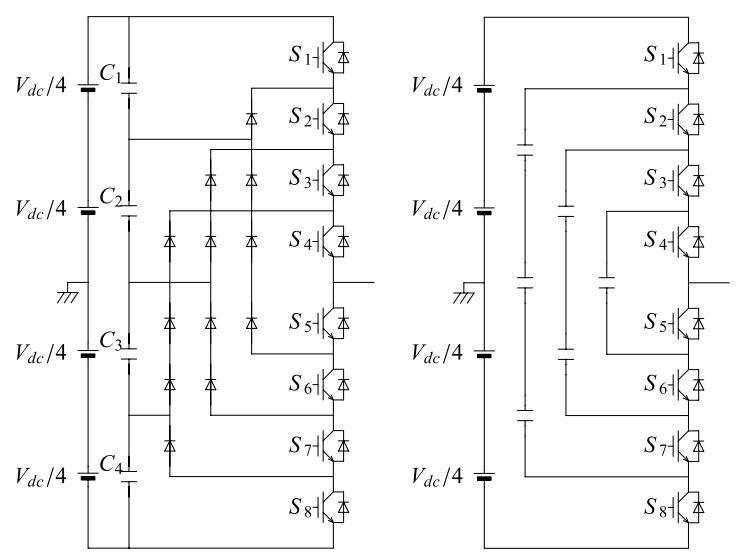

Fig. 1. Five-level clamped converters. (a) Diode clamped and (b) capacitor-clamped or flying-capacitor types

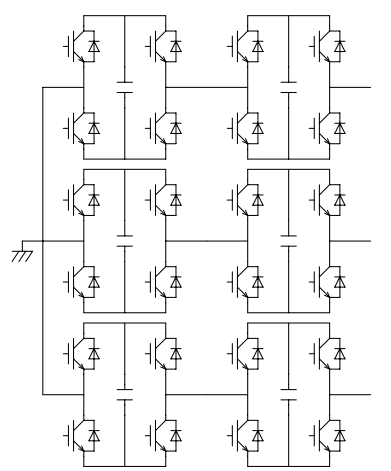

Fig. 2. Single-star bridge-cell configuration

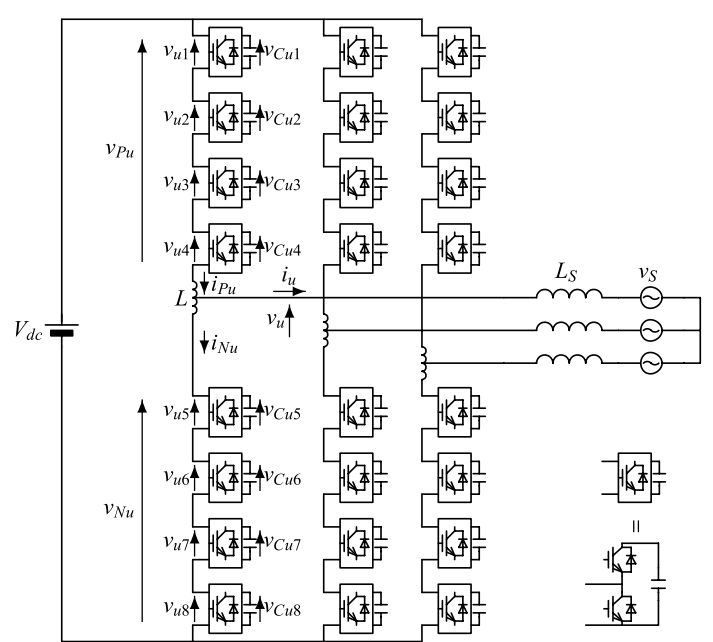

Fig. 3. Three-phase modular multilevel cascade converter (MMCC) based on double-star chopper cells (DSCC) 


\title{
Power System Stabilization Control by HVDC with SMES Using Virtual Synchronous Generator
}

\author{
Zarchi Linn Non-member (Osaka University, linn@pe.eei.eng.osaka-u.ac.jp) \\ Yushi Miura Member (Osaka University, miura@eei.eng.osaka-u.ac.jp) \\ Toshifumi Ise Senior Member (Osaka University, ise@eei.eng.osaka-u.ac.jp)
}

Keywords: VSG, SMES, VSC-HVDC, synchronous generator, power fluctuation

The number of renewable power sources connected to electrical power grids, such as photovoltaics (PV) and wind turbines is rapidly increasing. With the expeditious increase in the capacity of distributed generators, maintenance of grid stability is becoming important because their extensive use in interconnected power grids tend to result in instability issues. Fluctuations in renewable energy can lead to system power oscillation which in turn results in instability and frequency deviation problems in power systems. To preserve the positive effect of inertia on stability of power systems, methods for allowing an inertial response need to be developed. In this study, VSC-HVDC (high-voltage DC system that uses a voltage source converter) connected with a weak AC system is studied. SMES (superconducting magnetic energy storage) is used in the DC link of the HVDC system to compensate for power fluctuation in order to maintain a constant power flow in one terminal side of HVDC. Recently, a novel concept VSG (virtual synchronous generator) control function was proposed as a control strategy to overcome stability issues in power systems. However, the use of the VSG method in the stabilization of large power systems is uncommon. Therefore, in this paper, we propose a VSG control method that is based on the theory of synchronous generators (SG) for grid stabilization. Furthermore, we describe conventional speed deviation control of the grid side to regulate the frequency to be closest to its nominal level. The proposed system is shown in Fig. 1.

In the VSG control concept, rectifier side converters are controlled to behave like a SG. The control scheme of VSG is based on the swing equation (1) for SGs.

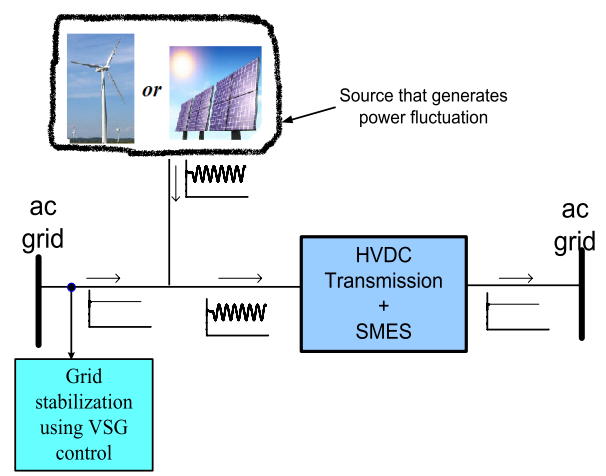

Fig. 1. System layout

$$
P_{g 0}-P_{g}=M \frac{d}{d t} \omega_{m}-D \Delta \omega_{m} \ldots \ldots \ldots \ldots \ldots \ldots \ldots \ldots \ldots \ldots \ldots(1)
$$

where $P_{g 0}$ and $P_{g}$ are the input and output power of SG, respectively, $D$ is the damping coefficient, and $\omega_{m}$ is the virtual rotating frequency. The proposed control block of VSG is shown in Fig. 2. In this figure, SG represents an AC grid while disturbance source represents a distributed generator. In VSG control, the virtual mechanical phase $\left(\theta_{m}\right)$ is controlled as a phase reference to generate a three-phase sinewave for rectifier AC voltage.

Although both VSG and speed deviation control can stabilize grid power to avoid the influence of disturbance, VSG control is more convenient because it can maintain the nominal value of grid frequency without requiring speed information from a generator. Because there is virtual inertia that can revert the frequency to its nominal value in VSG, it can contribute to increase the natural inertia response of the system.

To investigate the performance of the proposed control system for grid stabilization and the effectiveness of SMES for compensation of power fluctuation, simulations were carried out using PSCAD/EMTDC.

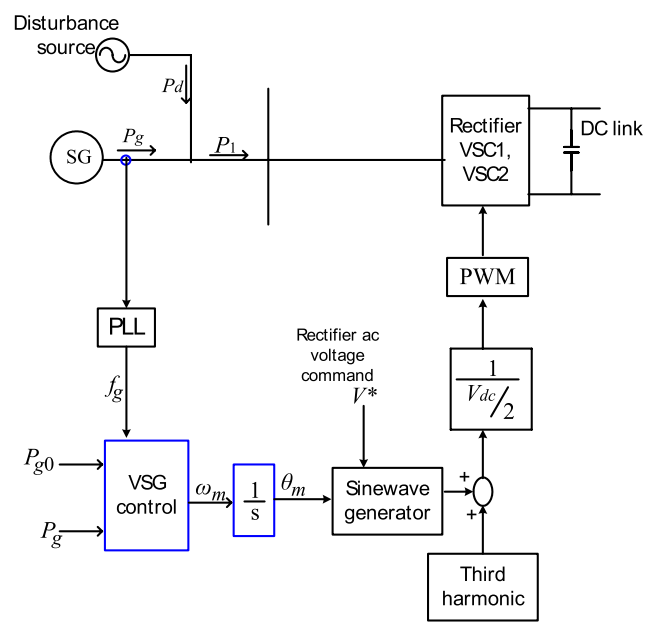

Fig. 2. VSG control block 


\title{
3-D Finite Element Analysis of Linear Resonant Actuator under PID Control Using Back EMF
}

\author{
Yasuyoshi Asai Student Member (Osaka University, yasuyoshi.asai@ams.eng.osaka-u.ac.jp) \\ Katsuhiro Hirata Senior Member (Osaka University, k-hirata@ams.eng.osaka-u.ac.jp) \\ Tomohiro Ota Member (Panasonic Electrics Works, Ltd., ota.tomorrow@jp.panasonic.com)
}

Keywords: linear resonant actuator, PWM control, PID control, finite element method, dynamic analysis

Recently, linear resonant actuators (LRAs) have been used in a wide range of applications, for example, electrical shavers, electric toothbrushes, air compressors, and artificial hearts, since they have many advantages: high efficiency, simple structure, easy control, and so on. On the other hand, they have a problem in that the amplitude decreases significantly in response to an external load. To overcome this problem, pulse width modulation (PWM) feedback control is adopted, in which the back electromotive force (EMF) of the coil is detected to determine the current duty cycle. However, it has been found that it is difficult to maintain constant amplitude by using a control function involving the determination of the current duty cycle. Therefore, more effective feedback control is investigated: PID control in PWM feedback control is used to keep the amplitude constant against an external load.

In this paper, we propose a numerical analysis method involving the use of the 3-D FEM for predicting the dynamic characteristics of an LRA under PID control in PWM feedback control. The effectiveness of the method is verified through a comparison of the prediction with measurements.

The basic structure of the LRA considered in this study is shown in Fig. 1. This actuator mainly consists of two movers, a common stator, and resonance springs that maintain an air gap. The two movers move in opposite directions when the coil is excited. This actuator is operated under PWM feedback control, and the current duty cycle is determined by detecting the back EMF when the coil is not excited.

Fig. 2 shows unipolar PWM feedback control of this actuator. First, in interval (a), the coil detects the maximum back EMF $V_{1}$ after the back EMF is reduced to zero. In interval (b), the coil shows a time delay. In interval (c), the extent of coil excitation is according to the current duty cycle determined from $V_{1}$. In interval (d), the current circulates through a diode. Finally, in interval (e), the circuit is opened.

Fig. 3 shows a plot of the computed and measured values of the amplitude and the average current against the load. Since it is difficult to maintain a constant amplitude, the use of more effective feedback control involving the use of PID control in PWM feedback control to maintain the constant amplitude against an external load is investigated.

Under unipolar PWM feedback control, the coil is excited in interval (c) by the control function. Here, the current duty cycle is determined under PID control by using $V_{1}$.

Fig. 4 shows the computed and measured values of the amplitude and average current when the LRA is operated with an external load under PID feedback control. As can be seen, the computed and measured values are in good agreement. Moreover, the amplitude is kept constant under a load of about $0.8 \mathrm{~N}$ by PID control. For loads greater than $0.8 \mathrm{~N}$, the amplitude decreases because the current duty cycle becomes nearly $100 \%$. These results show the effectiveness of feedback control using PID control.

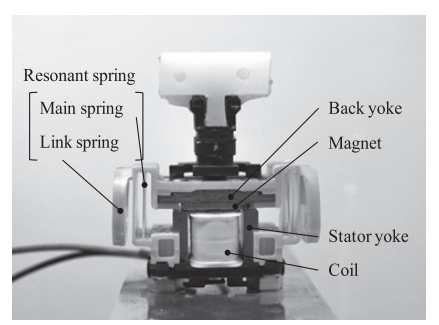

Fig. 1. Basic structure of the LRA

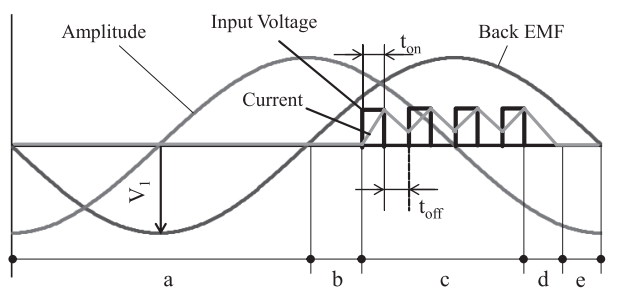

Fig. 2. Unipolar PWM feedback control

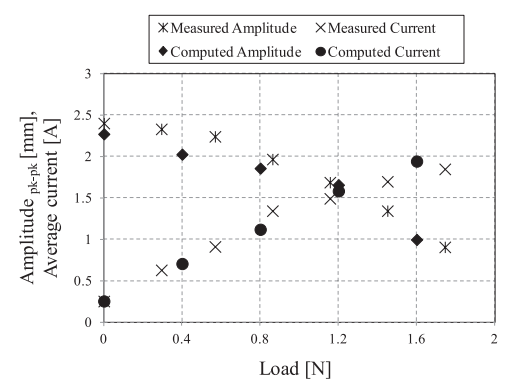

Fig. 3. Load characteristics under conventional control

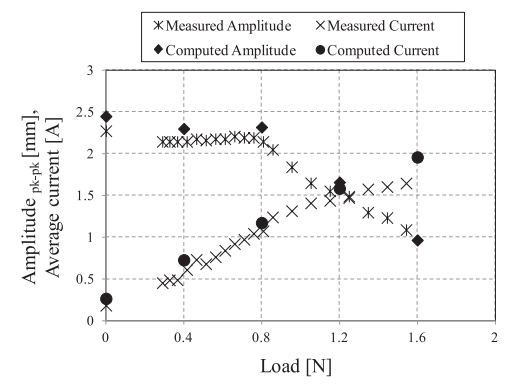

Fig. 4. Load characteristics under PID control 


\title{
Two-DOF Resonant Actuator Using Vector Control
}

\author{
Yasuyoshi Asai Student Member (Osaka University, yasuyoshi.asai@ams.eng.osaka-u.ac.jp) \\ Katsuhiro Hirata Senior Member (Osaka University, k-hirata@ams.eng.osaka-u.ac.jp) \\ Tomohiro Ota Member (Panasonic Electrics Works, Ltd., ota.tomorrow @jp.panasonic.com)
}

Keywords: dynamic analysis, resonant actuator, finite element method, vector control, multi-degree-of-freedom

Linear resonant actuators (LRAs) have been used in a wide range of applications because they are capable of short-stroke reciprocating motion despite their compact size and small weight. Recently, with expansion of their application field, it has necessary to drive actuators with a degree of freedom of two or more. We have proposed actuators that can drive multi-degree-of-freedom systems. We have also proposed a method involving a combination of a magnetic field, an electric field, a control method, and motion equation employing our 3-D FEM code for analyzing the dynamic characteristics of resonant actuators.

The actuator that has been studied so far is a model that is operated under open control, and the model with the drive axis in the direction of the air gap has not been examined. While the range of movement of the latter model in the direction of the air gap is limited, a larger thrust can be obtained compared to the thrust in the horizontal direction. Moreover, the examination of this model is important for the development of a multi-degree-of-freedom actuator.

This paper proposes a new two-degree-of-freedom (two-DOF) resonant actuator whose drive axis is in the direction of the air gap, and a control method for the actuator. The effectiveness of this actuator and the control method was verified by 3D finite element analysis.

The basic structure of the two-DOF resonant actuator is shown in Fig. 1. This actuator mainly consists of a mover, a stator, and resonant springs in the $\mathrm{X}$ and $\mathrm{Z}$ directions that support the mover to maintain the airgap $(1 \mathrm{~mm})$. The mover consists of permanent magnets $(\mathrm{NbFeB}, \mathrm{Br}=1.42 \mathrm{~T})$ fixed on the back yoke $(\mathrm{SUY})$. The stator comprises an E-type laminated yoke with three 45-turn excitation coils. This actuator is assumed to move $\pm 1.2 \mathrm{~mm}$ in the $\mathrm{X}$-direction and $\pm 0.5 \mathrm{~mm}$ in the Z-direction.

Vector control is employed to control each drive axis independently; the field current element ( $i_{d}$ element) is used as the Z-axis thrust element and the torque current element ( $i_{q}$ element) is used as the $\mathrm{X}$-axis thrust element.

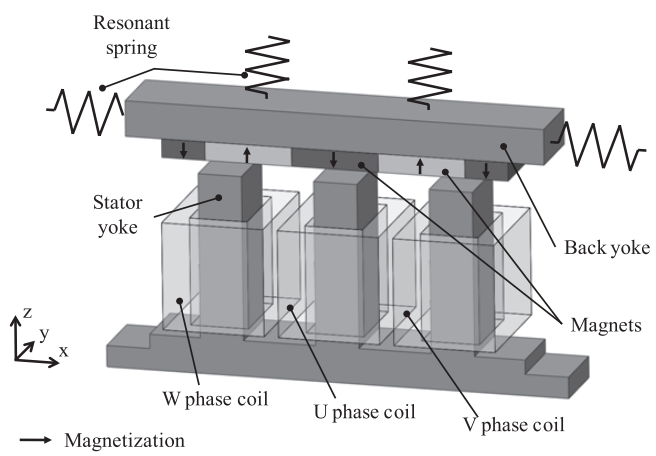

Fig. 1. Basic structure of the two-DOF resonant actuator
Fig. 2 shows the analysis results for both oscillation amplitudes of the mover when the actuator is operated to resonate in the direction of each axis under vector control. The resonant frequencies in the directions of the $\mathrm{X}$ and $\mathrm{Z}$ axes are $224 \mathrm{~Hz}$ and $422 \mathrm{~Hz}$, respectively. As can be seen from these results, the mover can be independently controlled along each axis.

Fig. 3 shows the analysis results for both oscillation amplitudes of the mover when the actuator is operated to simultaneously resonate in the direction of both $\mathrm{X}$ and $\mathrm{Z}$ axes under vector control. As can be seen, the mover can be simultaneously driven along each axis.

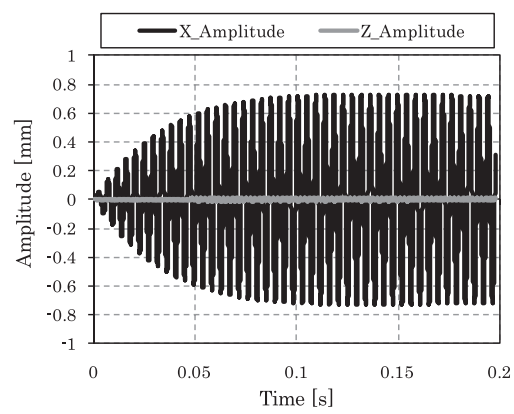

(a) $\mathrm{X}$-axis drive

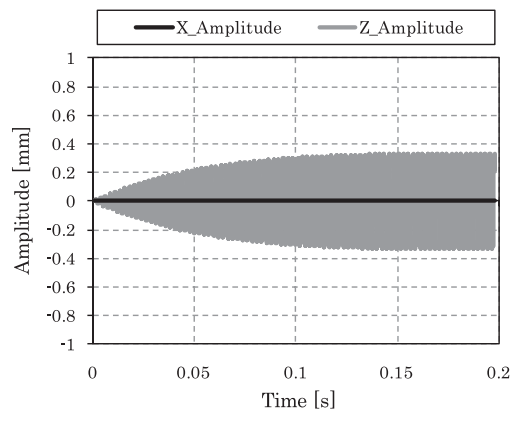

(b) Z-axis drive

Fig. 2. Transient characteristics (single drive)

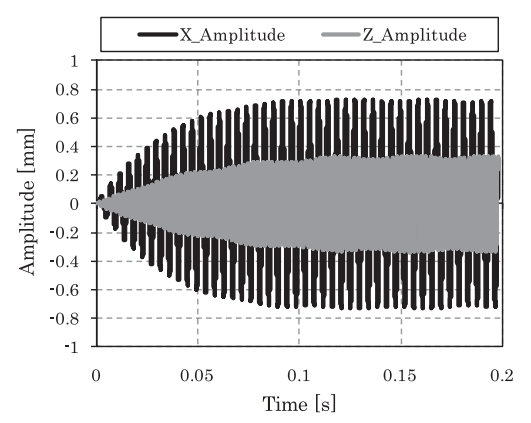

Fig. 3. Transient characteristics (biaxial drive) 